\title{
Precision spectroscopy using the Lamb dip in a pure ion plasma
}

P. N. Barnes

paul.n.barnes@us.army.mil

Grant W. Hart

Follow this and additional works at: https://scholarsarchive.byu.edu/facpub

Part of the Mathematics Commons

\section{Original Publication Citation}

Barnes, P. N. and G. W. Hart. "Precision spectroscopy using the Lamb dip in a pure ion plasma."

Review of Scientific Instruments 64 (1993): 579-58.

\section{BYU ScholarsArchive Citation}

Barnes, P. N. and Hart, Grant W., "Precision spectroscopy using the Lamb dip in a pure ion plasma" (1993). Faculty Publications. 710.

https://scholarsarchive.byu.edu/facpub/710 


\title{
Precision spectroscopy using the Lamb dip in a pure ion plasma
}

\author{
P. N. Barnes ${ }^{\text {a) }}$ and G. W. Hart \\ Department of Physics and Astronomy, Brigham Young University. Provo, Utah 84602
}

(Received 22 June 1992; accepted for publication 5 October 1992)

The use of the Lamb dip as a technique for precision spectroscopy in a non-neutral plasma is explored through computer modeling. Using singly ionized magnesium as the ion and under typical pure ion plasma conditions, the measurement appears to be feasible. Under the conditions calculated here, the Lamb dip is only $4 \%$ wider than the natural linewidth of the transition.

Pure ion plasmas have been used in recent years to make precise spectroscopic measurements on ions. ${ }^{1,2}$ The use of a pure ion plasma offers several advantages, such as long retention times for studying the ions $\mathrm{s}^{2-4}$ and the absence of free electrons, along with their effects. The low densities of the plasma mean ion-ion collisions can also be neglected. Usually, laser cooling has been used to cool the confined ions to milliKelvin temperatures. A disadvantage of this technique is that it requires two lasers, a cooling laser and an interrogation laser. In this note we study the possibility of using a single laser for precise spectroscopic measurements using the Lamb dip $^{5}$ to eliminate first-order Doppler broadening. Even though the accuracy of this technique is lower than that of laser cooling, the increased simplicity of the apparatus might make it attractive for some applications.

In this context, the Lamb dip arises in the laserinduced fluorescence from a plasma. If a laser beam which is tuned to a frequency slightly shifted from the resonant frequency of a transition is directed through a plasma and reflected back upon itself, there will be two groups of ions doppler shifted into resonance with the laser, one traveling in each direction. This will double the fluorescence from the ions. If, however, the laser is tuned exactly to the transition frequency, only one group of ions, those with velocity $v=0$, can respond and the fluorescence will decrease if the laser intensity is sufficient to saturate the transition. This decrease in signal is known as the Lamb dip.

We developed a computer model of this system, using the $3 p_{3 / 2} \rightarrow 3 s_{1 / 2}$ transition of ${ }^{24} \mathrm{Mg}^{+}$at $279.55 \mathrm{~nm}$, to see if such a technique was feasible in a pure ion plasma with reasonable experimental parameters. We solved the rate equations for the steady-state case, ignoring collisionally induced transitions and assuming a Maxwellian velocity distribution for the ions and Lorentzian line shapes for both the fluorescence line and the laser line. The fluorescence signal from the plasma is a function of the plasma's temperature, the intensity of the laser, and the linewidth of the laser. We have investigated the dip as a function of these parameters and give the results for a typical case.

Figure 1 shows the fluorescence expected as a function of laser frequency. The Lamb dip is clearly visible at the transition frequency. This figure is calculated for a laser "Current address: Wright Laboratory (POOC-3), Wright-Patterson
AFB, OH 45433-6563. intensity of $0.1 \mathrm{~W} / \mathrm{cm}^{2}$, with a laser bandwidth of $1 \mathrm{MHz}$ and an ion temperature of $900 \mathrm{~K}$. The relative depth of the dip (the dip depth divided by the maximum of the fluorescence curve) as a function of laser power is shown in Fig. 2. Not surprisingly, as the laser power increases and the transition becomes more saturated, the relative depth of the dip approaches 0.5. However, as also seen in Fig. 2, the relative width of the dip (the width of the dip normalized to the natural linewidth of the transition) also increases substantially due to saturation broadening as the laser power increases. The width of the dip does not continually increase as the laser power goes up, but is limited by the width of the Doppler profile. At higher powers than shown in this figure, the dip maintains about the same HWHM but decreases in depth.

The increasing width of the dip with higher power means that there is a tradeoff between the depth and width of the dip. For the conditions modeled here, a reasonable intensity would be $0.1 \mathrm{~W} / \mathrm{cm}^{2}$. This provides a relative dip depth of approximately 0.05 and a dip HWHM of 1.04 times the natural HWHM of the transition.

The required laser power and bandwidth are achievable using a cw dye laser pumped by an argon ion laser, frequency doubled to the neighborhood of $280 \mathrm{~nm}$. This could produce a laser power of about $1 \mathrm{~mW}$ with a bandwidth of about $1 \mathrm{MHz}$. With a 1-mm laser heam, we achieve an intensity of $0.1 \mathrm{~W} / \mathrm{cm}^{2}$. The other question is whether there is sufficient signal from a pure ion plasma to measure the dip.

A pure ion plasma can be contained in a modified Penning trap of the type used to confine pure electron plasmas. ${ }^{6}$ The confinement geometry, shown in Fig. 3, consists of a set of hollow cylindrical rings accurately aligned with an external magnetic field. The axial magnetic field provides the radial confinement for the ions, and axial confinement is provided by putting large positive potentials on the end rings. To allow the fluorescence signal out while maintaining the electrical boundary conditions at the wall, there would need to be a region at the center of the machine where the solid wall would be replaced by a metal mesh or a set of holes in the wall.

Because of the self electric field of the ions, there is a limit to the density that can be confined for a given magnetic field, called the Brillouin limit. ${ }^{7}$ For even fairly modest fields (less than $1 \mathrm{~T}$ ), a density of $1 \times 10^{12} \mathrm{~m}^{-3}$ is well below the limit, so it is a fairly conservative estimate to use 


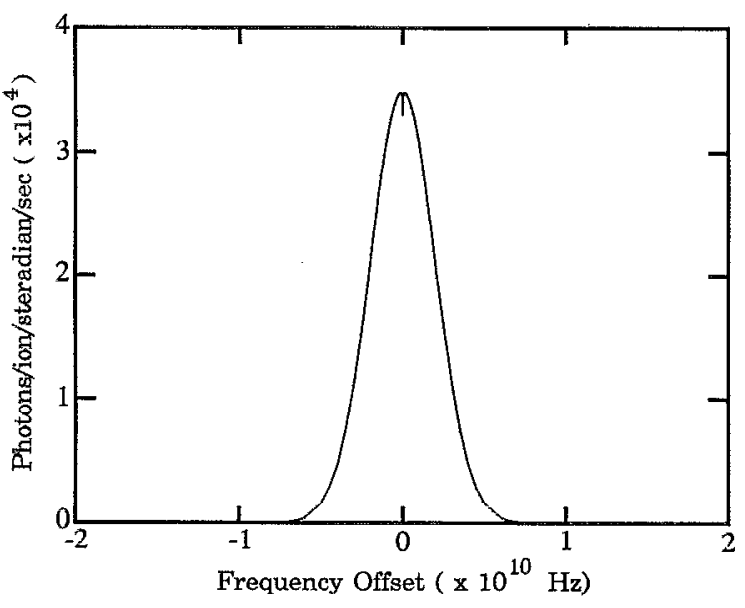

FIG. 1. The expected fluorescence from the $3 P_{3 / 2} \rightarrow 3 S_{1 / 2}$ transition of ${ }^{24} \mathrm{Mg}$ as a function of the laser frequency. The laser has an intensity of 0.1 $\mathrm{W} / \mathrm{cm}^{2}$ and a bandwidth of $1 \mathrm{MHz}$. The dip width is 1.04 times the natural linewidth of the transition.

that as the ion density. An experiment usually runs at magnetic fields several times this value ( $5 \mathrm{~T}$ might be typical) to enhance the confinement of the ions. It is also necessary in order to keep the gyroradius of the ions' orbit less than the 1-mm size of the laser beam.

The presence of the plasma's self electric field also requires that the laser beam pass down the symmetry axis of the plasma to minimize the Stark effect; along the axis, the self field is zero with a zero derivative. Another possible source of perturbing electric field occurs if the plasma is located off center within the cylindrical walls. In that case, an $l=1$ diocotron mode will exist in the plasma, which will create a small electric field on axis. This mode can easily be detected by the image charge it induces in the walls and it can be electronically damped to zero amplitude. ${ }^{8}$

Using low $f$-number optics (near $f$-1) and several fiber optic fibers aligned along the image of the laser beam,

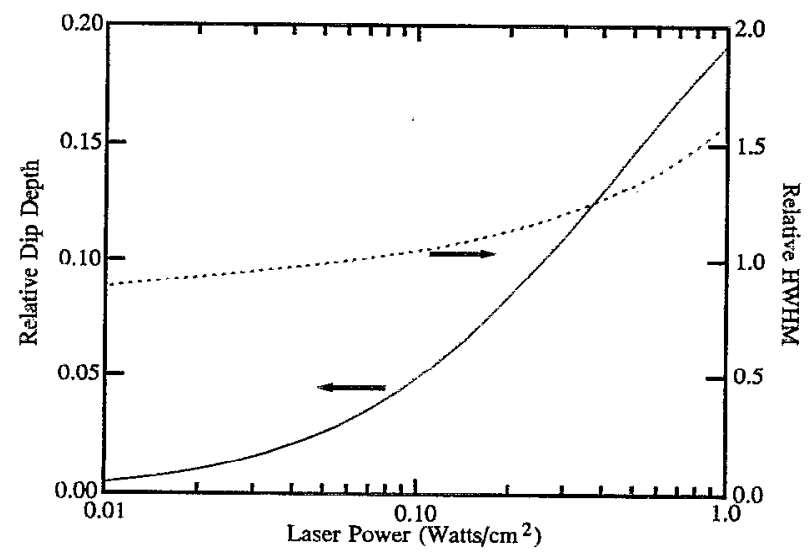

FIG. 2. The solid curve shows the relative depth of the dip as a function of laser power. The dashed curve shows the relative dip width as a function of laser power.

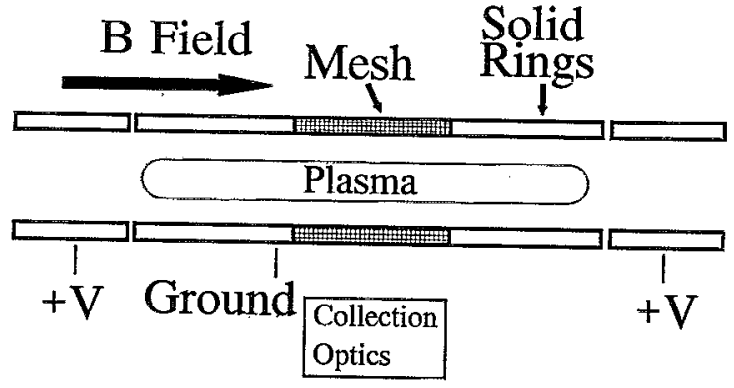

FIG. 3. Typical confinement geometry for a pure ion plasma experiment. The large potentials on the end rings provide axial confinement while the magnetic field provides the radial confinement. The mesh region in the center is to allow the fluorescence signal to get out while maintaining the necessary electrical boundary conditions.

it should be possible to collect a maximum fluorescence signal of about $40 \times 10^{6}$ photons/s. This means that the dip would be about $2 \times 10^{6}$ photons/s deep. Assuming no stray laser light, the noise that would confound the signal is the shot noise in the background fluorescence signal. This should be approximately equal to the square root of the number of photons collected. If the detection system has an integration time of about $1 \mathrm{~ms}$, the signal would be about 2000 photons, the background would be 40000 photons, and the noise would therefore be about 200 photons, giving a signal-to-noise ratio of 10 . Of course, longer integration times would give a better signal-to-noise ratio.

Observation times of the order of seconds should be easily obtainable in such a plasma. Pure electron plasmas have been confined for many minutes in this geometry, ${ }^{9}$ and a pure ion plasma should behave in a similar way.

Because of the low intensity of the laser beam, the ac stark broadening of the line will be negligibly small. Because the Lamb dip technique only selects for zero velocity in the direction of propagation of the laser beam, the second-order Doppler effect will still exist for the perpendicular velocity components. For an ion temperature of $900 \mathrm{~K}$ the second-order Doppler shift would be of the order of $3.7 \mathrm{kHz}$, compared to the natural linewidth of 43 $\mathrm{MHz}$.

'J. J. Bollinger, J. D. Prestage, W. M. Itano, and D. J. Wineland, Phys. Rev. Lett. 54, 1000 (1985).

${ }^{2}$ D. J. Larson, J. C. Bergquist, J. J. Bollinger, W. M. Itano, and D. J. Wineland, Phys. Rev. Lett. 57, 70 (1986).

${ }^{3}$ T. M. O'Neil, Phys. Fluids 23, 2216 (1980).

${ }^{4}$ T. M. O'Neil, Comments Plasma Phys. Controlled Fusion 5, 231 (1980).

${ }^{5}$ A. Szöke and A. Javan, Phys. Rev. Lett. 10, 512 (1963).

${ }^{6}$ J. S. DeGrassie and J. H. Malmberg, Phys. Rev. Lett. 39, 1077 (1977).

${ }^{7}$ R. C. Davidson, Theory of Nonneutral Plasmas (Benjamin, Reading, MA, 1974), p. 4.

${ }^{8}$ W. D. White, J. H. Malmberg, and C. F. Driscoll, Phys. Rey. Lett. 49, 1822 (1982); K. S. Fine, Ph. D. Dissertation, University of California, San Diego, 1988.

${ }^{9}$ C. F. Driscoll, K. S. Fine, and J. H. Malmberg, Phys. Fluids 29, 2015 (1986). 